\title{
Computationally EFFicient Two Stage SEQUENTIAL FRAMEWORK FOR STEREO MATCHING
}

\author{
${ }^{1}$ Bindu N.S and ${ }^{2}$ H.S.Sheshadri \\ ${ }^{1}$ Vidyavardhaka college of Engineering, Dept of E\&C, Mysore, India \\ ${ }^{2}$ PES College of Engineering, Dept of E\&C, Mandya, India
}

\begin{abstract}
:
Almost all the existing stereo algorithms fall under a common assumption that corresponding color or intensity values will be similar like one another. On the other hand, it is also not that true in practice where the image color or intensity values are regularly affected by different radiometric factors like illumination direction, change in image device, illuminant color and so on. For this issue, the information about the raw color of the images which is recorded by the camera should not depend on it totally, and also the common assumptions on color consistency doesn't influence good (great) between the stereo images in real scenario. Therefore, most of the conventional stereo algorithms can be seriously degraded in terms of performance under radiometric variations. In this work, we intend to develop a new stereo matching algorithm which will be insensitive to change in radiometric conditions between stereo pairs i.e. left image as well as right image. Unlike the other stereo algorithms, we propose a computationally efficient two stage sequential framework for stereo matching which can handle the various radiometric variations between the stereo pairs.Experimental results proves that the proposed method outperforms extremely well compare to other state of the art stereo methods under change in various radiometric conditions for a given stereo pair and it is also found from the results that the execution time is less compare to existing methods.
\end{abstract}

\section{KEYWORDS:}

Stereo matching, radiometric variation, rank transform, bilateral filter, illumination, exposure.

\section{INTRODUCTION}

The aim of the stereo matching is to get better 3D information about a scene just by analyzing and finding the appropriate correspondence amongst the images that have been captured from different viewpoints.However, finding accurate correspondence in a given image is not going to be an easy task owedtoo many constraints like occluded regions, discontinuity in object boundaries, textureless regions, illumination and many other problems during the matching process. These are some of the constraints which takesa challenging task in research and also the research is going on since many decades.Effort towards this challenging area have given a great resultsby taking inputs from the Middlebury dataset [1], [2].

Various factors avoids the two corresponding pixel value of an image having a very same color pixel value. Radiometric change i.e. illumination and occlusion is the major andpreventing aspect, which includes the camera device variation for different stereo pair, lighting geometry, change in illuminant color [3]. A distinct pixel values i.e. color values of an image can be achievedby viewing the same scene many times as much as possible. The main purpose is that, at all times the intensity on any point possibly will be determined by the direction and angle in a Lambertian surface.By considering each and every point one can conclude that under the radiometric

DOI:10.5121/ijfcst.2016.6501 
variations, performance of existing stereo matching algorithms can be ruinedrigorously which can be seen in Fig.1.

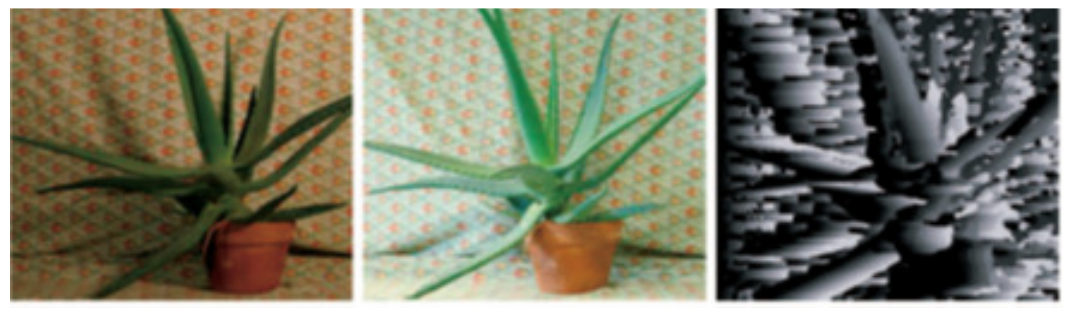

Fig.1. (a) Left image, (b) right image, (c) disparity (SAD+GC)

Keeping all of the above said points, an advancedand efficient stereo matching algorithm is developed which is capable of taking care of radiometric variations.

\section{RELATED WORK}

Data cost is the stereo information which plays a vital role on deciding the performance and quality of the output disparity [4]. Past from many decades it can be observed that most of the existing correlation based stereo matching algorithms [5] are more efficient under the ideal conditions but at the same time they are weak at change in radiometric variations. In [6], evaluation of various data cost function was carried out for better stereo matching by considering the change in radiometric conditions. On their study, local stereo algorithms like Sum of absolute difference (SAD), Sum of Squared Differences (SSD), Normalized cross-correlation (NCC), Zero mean sum of absolute differences (ZSAD), Sum of Hamming Distances (SHD) and many more algorithms very compared. Hirschmuller also compared Birchfield Tomashi (BT) data cost [7], Birchfield Tomashi with Laplacian of Gaussian (LOG) [8], Census transform and some of the semi global algorithms too. One of the most efficient data cost was BT, Where it is very insensitive due to camera sampling and at the same time it is also sensitive to change in radiometric conditions as it employs linearly interpolated function of intensity values. Due to the usage of second order derivatives, LOG filter is very insensitive in term of outliers but it is very sensitive to change in radiometric variations. One of the popular similarity measures is NCC [9] which is very much suitable for matching the affine transformed colored values, where it measures the angle of cosines between the matching vectors. This NCC algorithm as well as most of the local algorithms suffers from fattening effect which occurs across the object boundaries. In [10] Heo et al. proposed an algorithm which was based on log chromaticity normalization and adaptive normalized cross correlation (ANCC). The proposed method was very expensive and it was very weak due to global variations. In [11], Yong Seok Heo, Kyoung Mu Lee, proposed a new method which infers accuratedepth maps and color-consistent images between radiometrically varying stereo images, simultaneously. The proposed method was very accurate but again it was found that the execution time was more compare to other methods. In [12] Yong Seok Heo. Proposed a new method which was based on mutual information combined with SIFT descriptorto find correspondence for images which undergo local as well as global radiometric variations. Even though the proposed method was quite robust to radiometric variations it failed to overcome the execution time compare to our proposed method. The major drawback of most of the existing stereo matching algorithm was due to usage of the raw color information. This paper proposes a computationally efficient two stage sequential framework for stereo matching which can handle the various radiometric variations between the stereo pairs. 


\section{Proposed Block Diagram}

The proposed approach is shown in the Fig.2along with their individual constituent blocks. A stereo pair is taken as input from Middlebury dataset and the pre-processing step is followed and done. The complete explanation of each individual block is described and explained in the upcoming sections.

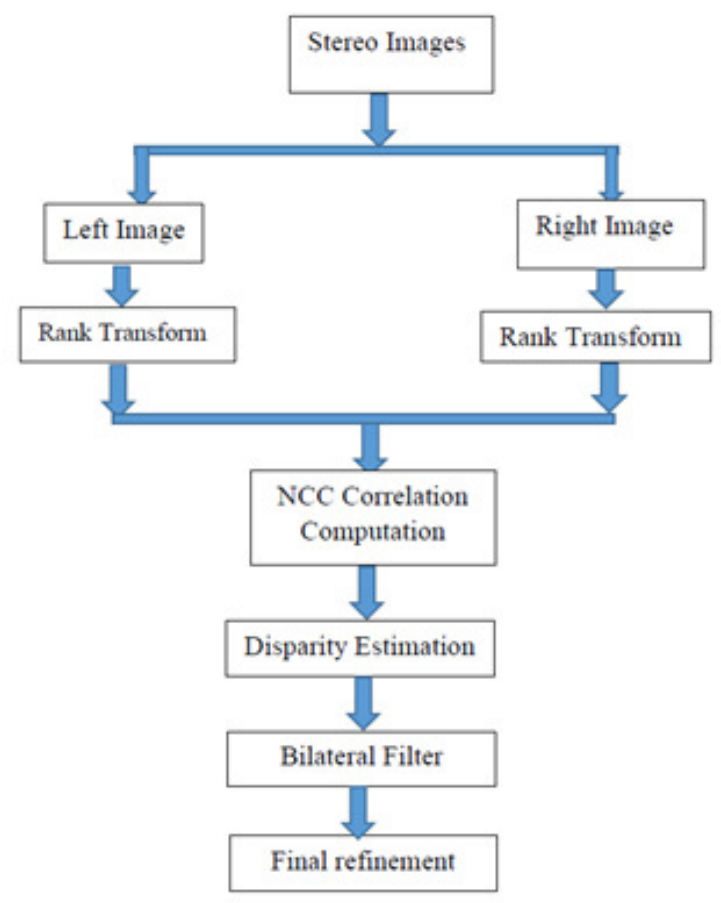

Fig2: Generalized Proposed block diagram

\subsection{RANK TRANSFORM (RT)}

Almost all the stereo matching algorithms which is existing till today, a transforms which are said to be non-parametric are used to search and find the possible pixel correspondence surrounded by the neighbor. The most regularly used transforms is theRT and CT. RT is susceptible to noise particularly when anarea which consist of no texture is considered, where it sets a pixel of the matching window as a center and also it takes a pixels in some other square neighborhood transform window. On the off chance that the middle pixel esteem not as much as neighborhood pixel, it returns 0 and 1 generally. In this way, the arrangement of pixels in reference image as well as target image turn into the arrangement of ordered pairs [13].

$$
E(P)=\bigcup_{P^{\prime} \in N(P)}\left(P^{\prime}, \xi\left(\boldsymbol{P}, \boldsymbol{P}^{\prime}\right)\right)
$$

Let $\mathrm{P}$ represents the pixel, $\mathrm{I}(\mathrm{P})$ be the intensity pixel, $\mathrm{N}(\mathrm{P})$ represents the square neighborhood which is of the diameter ' $d$ ' in turn surrounding $P$ and $E$ represents the exact reliance. $R(P)$ is not an intensity but it's rather an integer which has the range $\left\{0, d^{2}-1\right\}$ as 


$$
R(P)=\left\|\left\{P^{\prime} \in N(P) \mid I\left(P^{\prime}\right)<I(P)\right\}\right\|
$$

The key advantage of using this non-parametric transform called RT in stereo matching is it can reduce the effects of diverse gains and also bias camera, which is insensible to an image noise, further helps to improve the robustness to depth discontinuities.

\subsection{NCC CORRELATION COMPUTATION}

Correlation based techniques usually produces a dense map. This depth map can be obtained by simply calculating the disparity at every individual pixel with a pre-defined $3 \times 3$ neighborhood. The pre-defined location compared with target image in order to find a so called homologous pixel in that specified and pre-defined window. The main idea of using this NCC is to search and measure the pixels that are correlated within the stated range called ' $\mathrm{d}$ ' and this ' $\mathrm{d}$ ' ranging from (0...dmax). NCC is the correlation algorithm which is very frequently used and this NCC helps us to measure the so called matching contrast for different images [14, 15].

The generalized equation for an NCC is given in the equation (3).

$$
N C C=\frac{\sum(i, j) \in I_{1}(i, j) \cdot I_{2}(x+i, y+j)}{\sqrt[2]{\sum(i, j) \in w I_{1}^{2}(i, j) \cdot \sum(i, j) \in w I_{2}^{2}(x+i, y+j)}}
$$

Where $I_{1}(i, j)$ represents the left image and $I_{2}(x+i, y+j)$ represents the right image.

\subsection{DISPARITY}

The main aim of disparity is to search and identify the pixels values amongst the rectified images for the various stereo pair. The general expression to calculate the disparity between two images is given as

$$
d=x_{l}-x_{r}
$$

Where $x_{l}$ and $x_{r}$ indicates the left and right image at $\mathrm{x}$ - coordinate. Once the output of RT and NCC is obtained, the disparity estimation is done for various data sets.

\subsection{BILATERAL FiLTER (BF)}

Bilateral filtering (BT) aims at image smoothening and also preserves edge. This BF is used in texture relighting, denoising and texture editing. It has several advantages compare to other filters. The formulation of BT is very simple where each pixel will be replaced by the weighted average amongst its neighbors. In this work we intend to make use of this filter for better results and the general expression for BT is as follows

$$
I^{\text {filtered }}(x)=\frac{1}{W_{p}} \sum_{x_{i} \in \Omega} I\left(x_{i}\right) f_{r}\left(\left\|I\left(x_{i}\right)-I(x)\right\|\right) g_{s}\left(\left\|x_{i}-x\right\|\right)
$$

Where $I^{\text {filtered }}$ is the filtered image, I is the original image, $\Omega$ represents the window centered at $\mathrm{x}, f_{r}$ represents the kernel range which helps in smoothening differences amongst the intensities and $g_{s}$ represents the spatial kernel. 


\section{RESULTS AND DISCUSSION}

In this evaluation we consider a pair of left and right stereo image as well as its ground-truth disparity. These stereo pairs are taken from Middlebury stereo datasets [1,2]. The $d_{\max }$ i.e., disparity range is considered as ranging from 0 to 70 pixels. The main contribution of this work is to evaluate the robustness to change in illumination and exposure.

\subsection{Change In ILlumination}

In more or less all the papers, most of the authors have made an assumption saying that the corresponding values of pixels willhave the similar color values. But at the same time, the above held statement does not hold good for the case of stereo pair having different color pixel values. In this work, the exposure index value is set to 1 and vary theillumination index value from 1 to 3 with window size of 9x9. Fig.3 to Fig.6. Shows the output of ANCC, NCC, BT and proposed algorithm for various stereo pair Aloe, dolls, Moebius and cloth4. Fig.7 and Fig.8 shows the RMS and BAD pixel calculation.

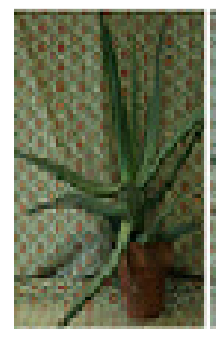

(a)

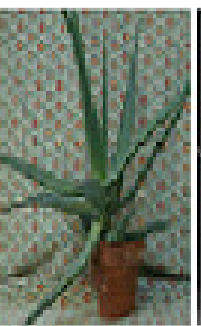

(b)

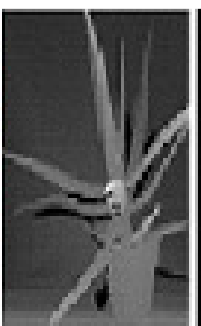

(c)

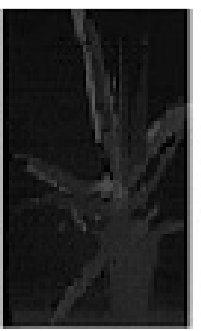

(d)

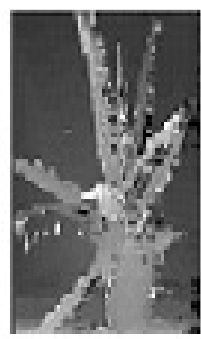

(e)

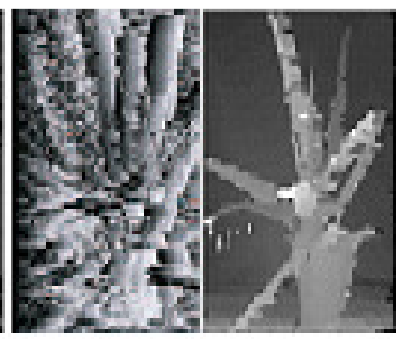

(f) (g)

Fig.3. Results of Aloe image pair by varying illumination. (a) The left image with illumination(1)exposure(1). (b) The right image with illumination(2)-exposure(1). (c) ground-truth (d) ANCC disparity (e) NCC (f) BT (g) Proposed algorithm

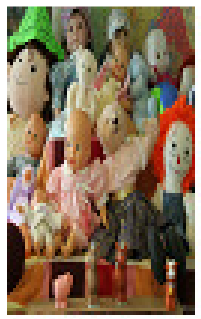

(a)

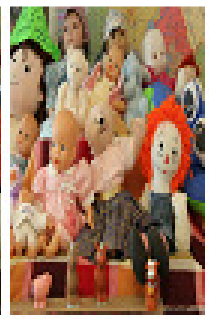

(b)

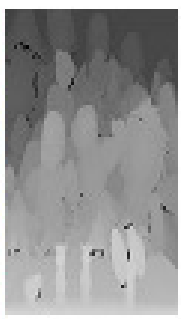

(c)

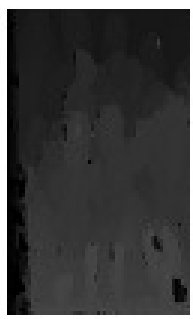

(d)

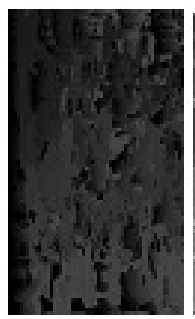

(e)

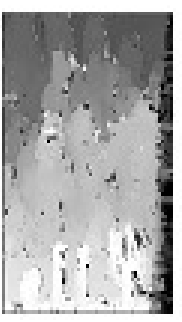

(f)

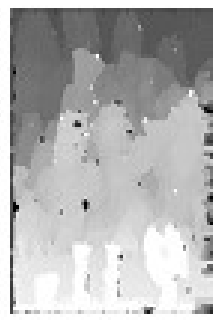

(g)

Fig4: Results of dolls image pair by varying illumination(a) The left image with illumination (1)-exposure (1). (b) The right image with illumination (2)-exposure (1). (c) ground-truth (d) ANCC disparity (e) NCC (f) BT (g) Proposed algorithm 
International Journal in Foundations of Computer Science \& Technology (IJFCST) Vol.6, No.5, September 2016

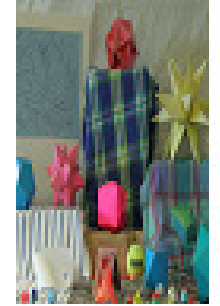

(a)

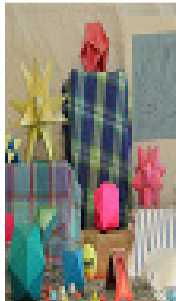

(b)

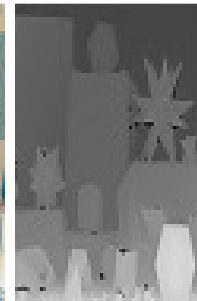

(c)

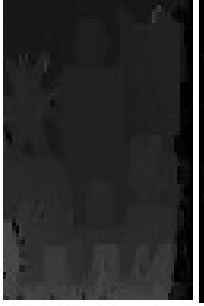

(d)

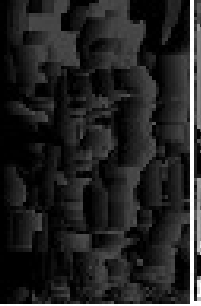

(e)

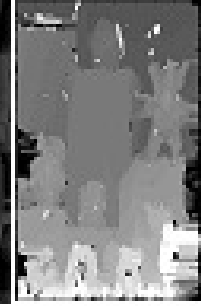

(f)

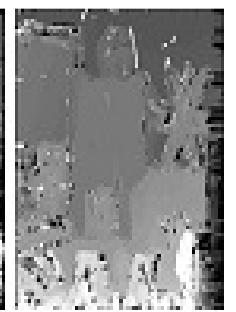

(g)

Fig5: Results of Moebius image pair by varying illumination. (a) The left image with illumination (1)exposure (1). (b) The right image with illumination (2)-exposure (1). (c) ground-truth (d) ANCC disparity (e) NCC (f) BT (g) Proposed algorithm

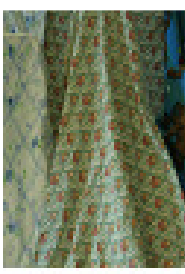

(a)

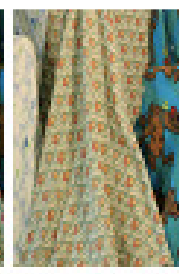

(b)

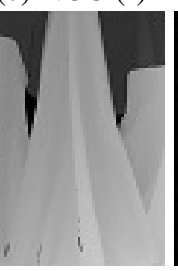

(c)

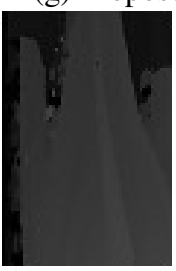

(d)

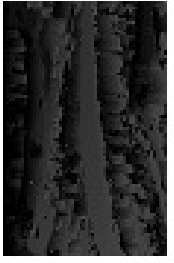

(e)

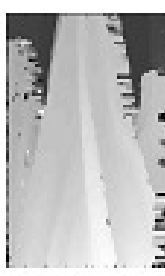

(f)

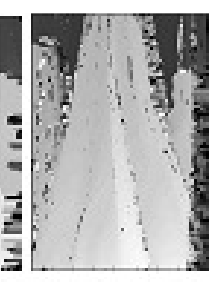

(g)

Fig.6: Results of cloth4 image pair by varying illumination. (a) The left image with illumination (1)exposure (1). (b) The right image with illumination (2)-exposure (1). (c) ground-truth (d) ANCC disparity (e) BT (f) Proposed algorithm (g) NCC

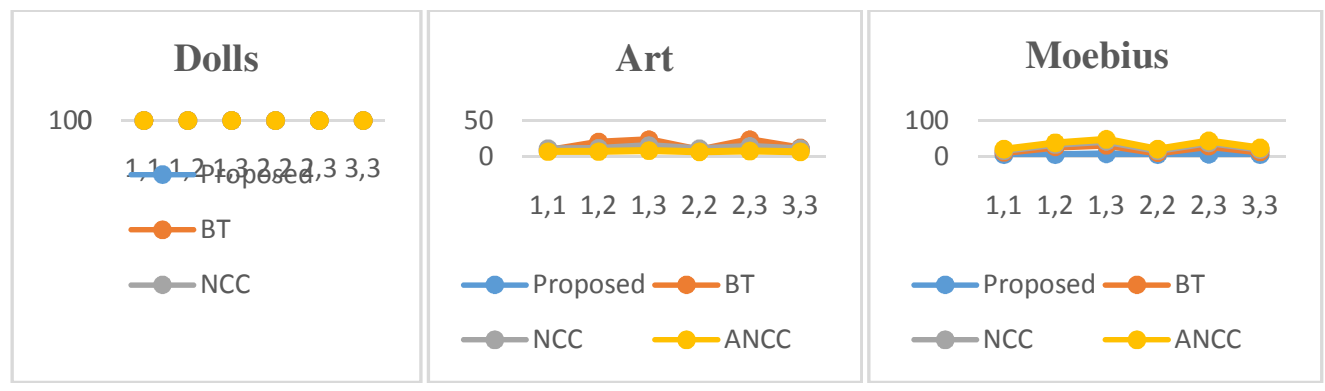

(a)

(b)

(c)

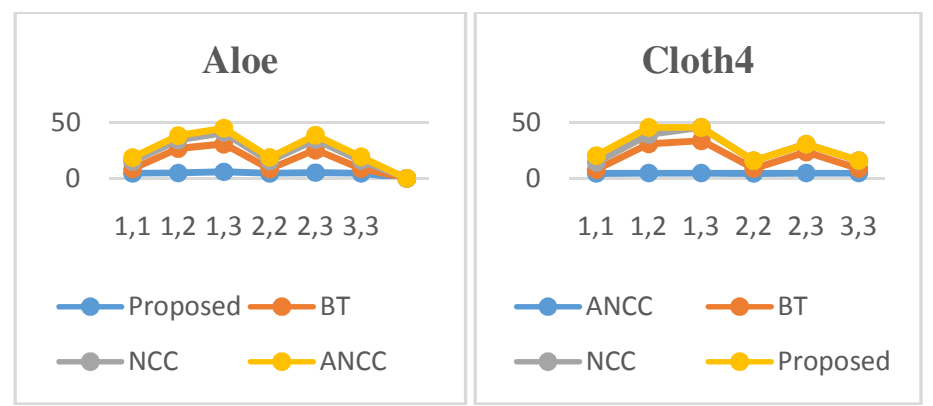

(d)

(e)

Fig.7: RMS calculationfor various stereo pairs with vary in illumination. 


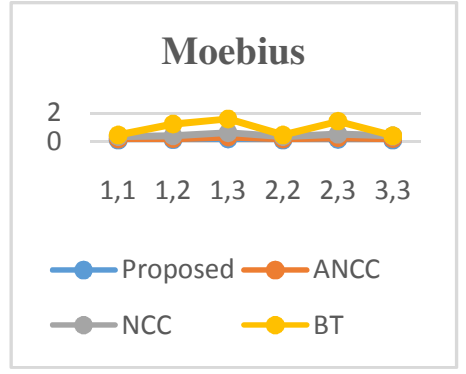

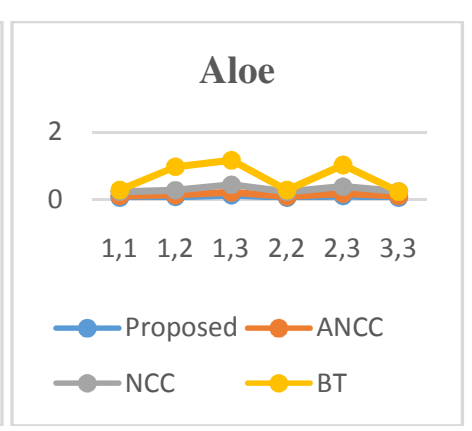

(c) (a)

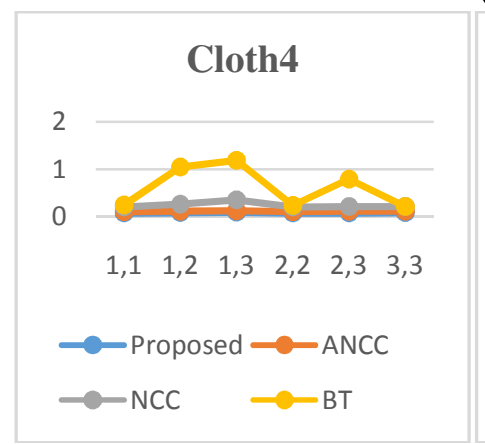

(d) (b)

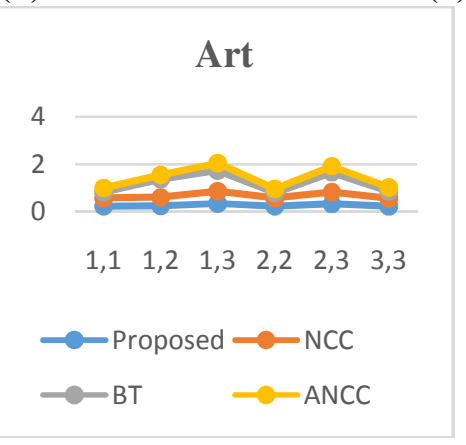

(e)

Fig.8: Bad pixel calculation for various stereopairs with vary in illumination

\subsection{Change In Exposure}

In this work, the illumination index value is set to 2 and vary theexposure index value from 0 to 2 with window size of 9x9. Fig.9 to Fig.12. Shows the output of ANCC, NCC, BT and proposed algorithm for various stereo pair Aloe, dolls, Moebius and cloth4. Fig.13 and Fig.14 shows the RMS and BAD pixel calculation.

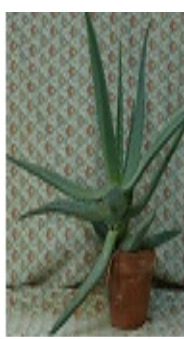

(a)

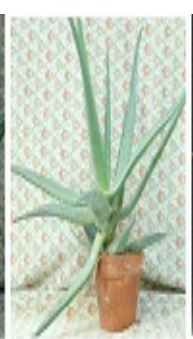

(b)

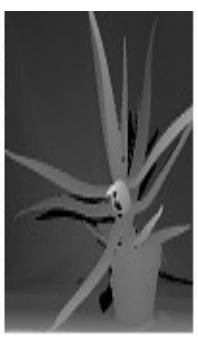

(c)

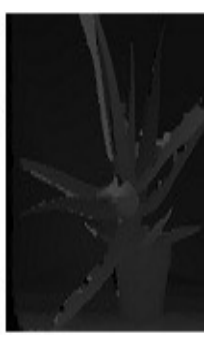

(d)

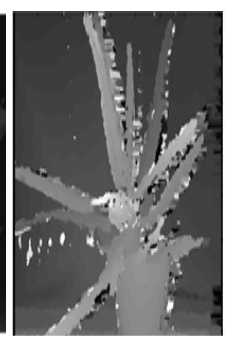

(e)

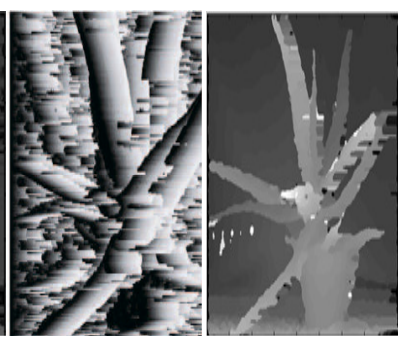

(f)

(g)

Fig.9: Results of Aloe image pair by varyingexposure (a) The left image with illumination (2)-exposure(1). (b) The right image with illumination(2)-exposure (2). (c) ground-truth (d) ANCC disparity (e) NCC (f) BT (g) Proposed algorithm 
International Journal in Foundations of Computer Science \& Technology (IJFCST) Vol.6, No.5, September 2016

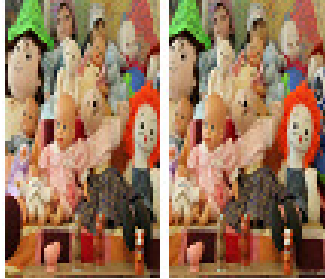

(a)

(b)

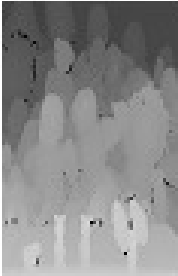

(c)

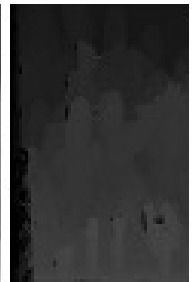

(d)

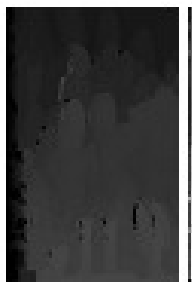

(e)

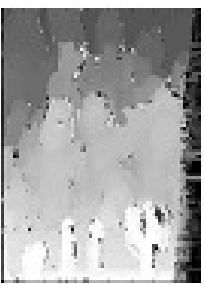

(f)

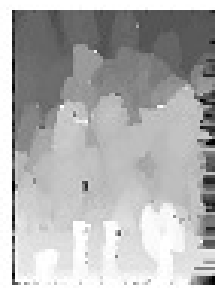

(g)

Fig.10: Results of doll image pair by varyingexposure. (a) The left image with illumination (2)exposure(1). (b) The right image with illumination(2)-exposure (2). (c) ground-truth (d) ANCC disparity (e) BT (f) NCC (g) Proposed algorithm

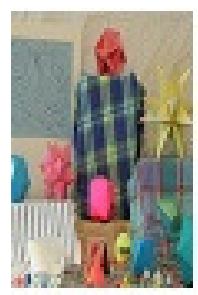

(a)

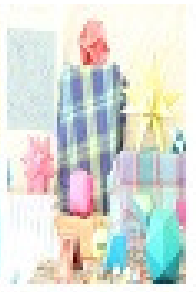

(b)

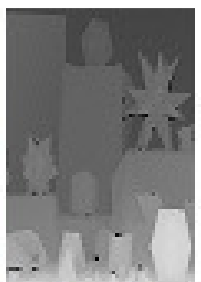

(c)

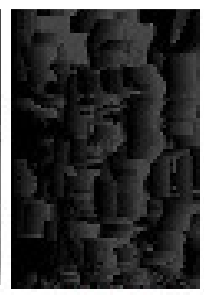

(d)

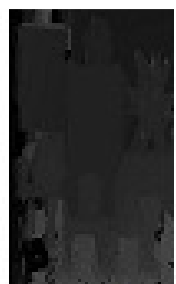

(e)

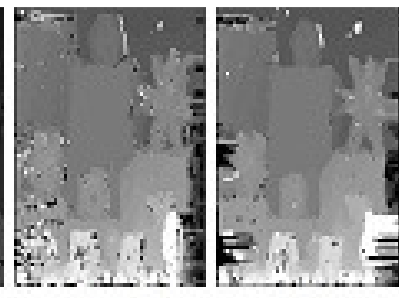

(f)

(g)

Fig.11: Results of Moebius image pair by varyingexposure(a) The left image with illumination (2)exposure(1). (b) The right image with illumination(2)-exposure (2). (c) ground-truth (d) BT disparity (e) ANCC (f) NCC (g) Proposed algorithm

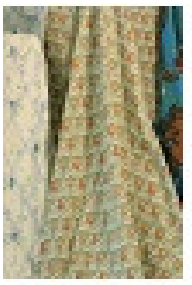

(a)

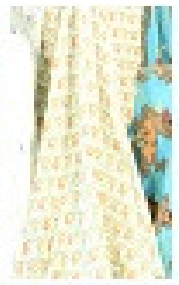

(b)

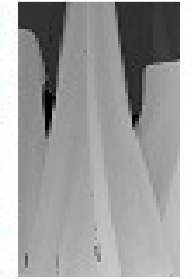

(c)

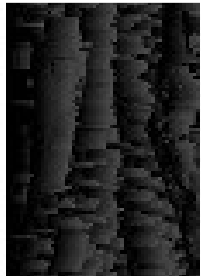

(d)

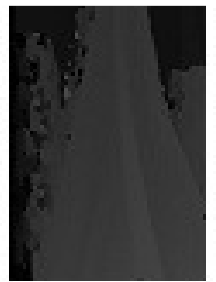

(e)

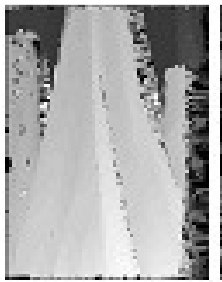

(f)

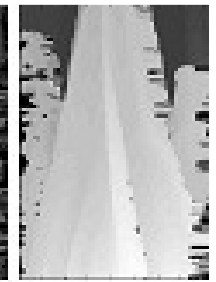

(g)

Fig.12: Results of Cloth4 image pair by varyingexposure. (a) The left image with illumination (2)exposure(1). (b) The right image with illumination(2)-exposure (2). (c) ground truth (d) BT disparity (e) ANCC (f) NCC (g) Proposed algorithm

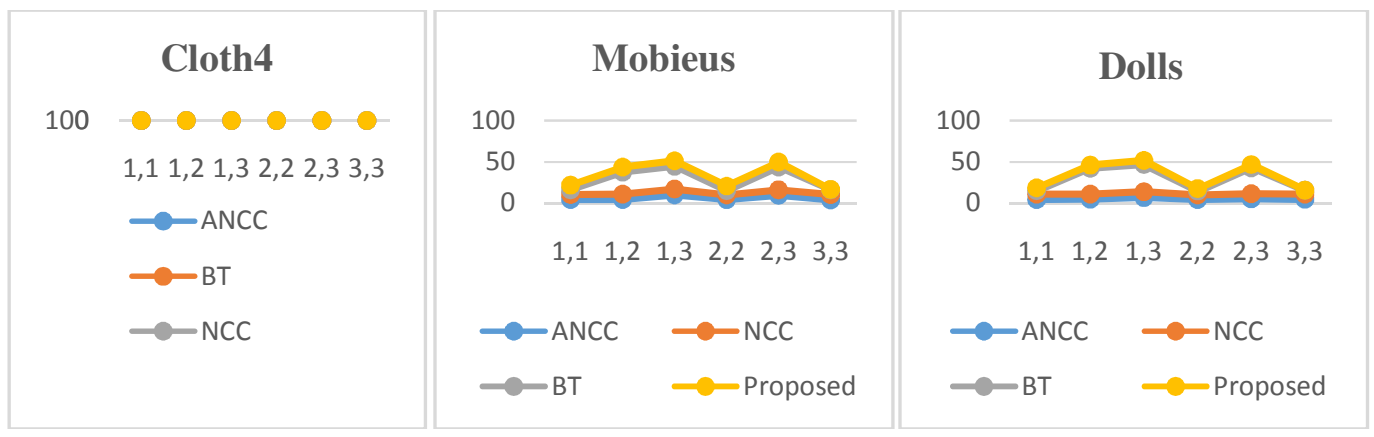

(a) (b) (c) 
International Journal in Foundations of Computer Science \& Technology (IJFCST) Vol.6, No.5, September 2016

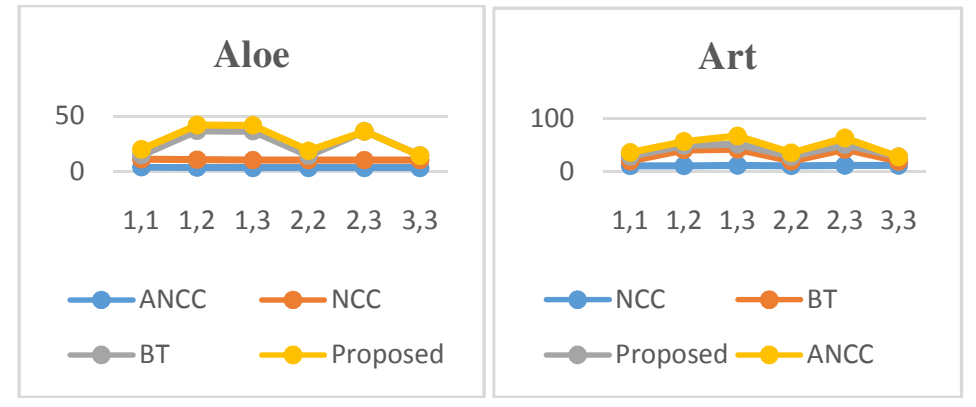

(d)

(e)

Fig.13: RMS calculation for variousstereo pairs with vary in exposure.

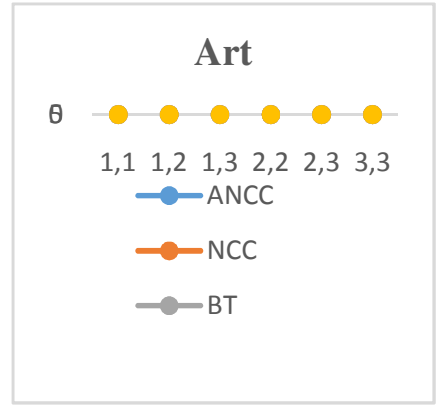

(a)

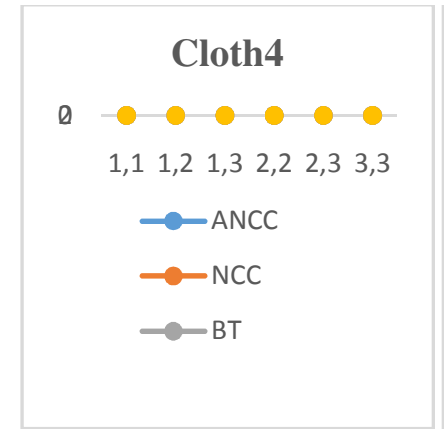

(b)

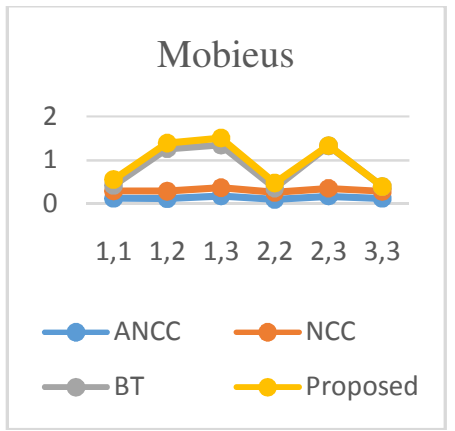

(c)

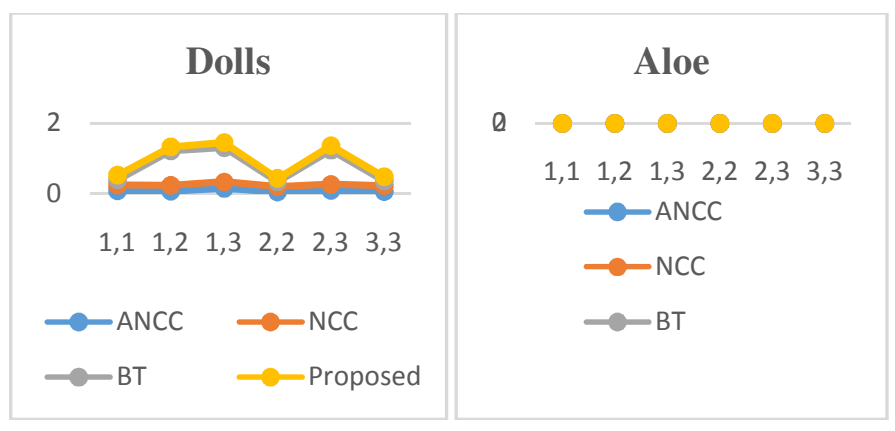

(d)

(e)

Fig.14: Bad pixel calculation for variousstereo pairs with vary in exposure..

\section{CONCLUSION}

As far as real time applications are considered, the correlation based stereo algorithms play an important roleas it is one of the most popular and very efficient and effective algorithms. In this work we have considered 3 correlation based stereo algorithms namely ANCC, NCC and BT and also analysis has been done considering various parameters. It is then compared with the proposed algorithm by using a window size of 9 . The evaluation is done towards the robustness to change in illumination and exposure in our algorithm. It is also very important and necessary to get the better results due to radiometric changes.As a key solution to many of the above stated problems, an efficient hybrid correlation of rank transform, NCC followed by bilateral filter is 
developed for better result and also selection of suitable window size plays an important role in deciding the final disparity.

\section{REFERENCE}

[1] http://vision.middlebury.edu/stereo/, 2010

[2] D. Scharstein and R. Szeliski, "A Taxonomy and Evaluation of Dense Two-Frame Stereo Correspondence Algorithms," Int'1 J.Computer Vision, vol. 47, no. 1, pp. 7-42, 2002.

[3] Y.S. Heo, K.M. Lee, and S.U. Lee, "Illumination and Camera Invariant Stereo Matching," Proc. IEEE Conf. Computer Vision andPattern Recognition, 2008.

[4] H. Hirschmuller and D. Scharstein, "Evaluation of stereo matching costs on images with radiometric differences," IEEETransactions on Pattern Analysis and Machine Intelligence,vol. 31, no. 9, pp. 1582-1599, 2009.

[5] D. Scharstein and R. Szeliski, "A taxonomy and evaluation ofdense two-frame stereo correspondence algorithms," Journal of Computer Vision, vol. 47, no. 1-3, pp. 7-42, 2001.

[6] Raghavendra U, KrishnamoorthiMakkithaya, Karunakar A. K,Qualitative and Quantitative Evaluation of Correlation Based StereoMatching Algorithms, Lecture Notes in Computer Science, SpringerBerlin/Heidelberg, International Conference on Advanced Computing,Networking and Security, pp. 242- 252, 16th Dec 2011, NITK,Surathkal.

[7] S. Birchfield and C. Tomasi, "A Pixel Dissimilarity Measure that Is Insensitive to Image Sampling," IEEE Trans. Pattern Analysis and Machine Intelligence, vol. 20, no. 4, pp. 401-406, Apr. 1998.

[8] H. Hirschmuller, P. Innocent, and J. Garibaldi, "Real-Time Correlation-Based Stereo Vision with Reduced Border Errors," Int'1 J. Computer Vision, vol. 47, nos. 1-3, pp. 229-246, 2002.

[9] O. Faugeras, B. Hotz, H. Mathieu, T. Viville, Z. Zhang, P. Fua, E. Thron, and P. Robotvis, "Real time correlationbased stereo: Algorithm, implementations and applications," Technical Report RR2013 INRIA, 1996.

[10] Y. S. Heo, K. M. Lee, and S. U. Lee, "Robust stereo matchingusing adaptive normalized crosscorrelation", IEEE Transactions on Pattern Analysis and Machine Intelligence, vol. 33, no. 4, pp. 807-822, 2011.

[11] Yong Seok Heo, Kyoung Mu Lee, and Sang Uk Lee "Simultaneous Color Consistency and Depth Map Estimation for Radiometrically Varying Stereo Images", IEEE 12th International Conference on computer vision, vol 31, no.9, pp. 1771 - 1778, 2009.

[12] Yong Seok Heo, Kyoung Mu Lee, and Sang Uk Lee "Mutual Information-based Stereo Matching Combined with SIFT Descriptor in Log-chromaticity Color Space", IEEE Conference on computer vision and pattern recognition, vol 31, no.9, pp.445 - 452, 2009

[13] Scharstein, D., \& Szeliski, R. (2002). A taxonomy and evaluation of dense two-frame stereo correspondence algorithms. International Journal of Computer Vision, 47, 7-42.

[14] O. Faugeras, B. Hotz, H. Mathieu, T. Vie'ville, Z. Zhang, P. Fua, E. The'ron, L. Moll, G. Berry, J. Vuillemin, P. Bertin, and C. Proy, "Real Time Correlation-Based Stereo: Algorithm, Implementations and Applications," Technical Report RR-2013, INRIA, 1993.

[15] M. Goesele, N. Snavely, B. Curless, H. Hoppe, and S.M. Seitz, "Multi-View Stereo for Community Photo Collections,” Proc. IEEE Int'l Conf. Computer Vision, 2007. 